\title{
Exploring the Influence of Critical Parameters for the Effective Synthesis of High-Quality 2D-MXene
}

Sana Munir ${ }^{\mathrm{a}}$, Aamir Rasheed ${ }^{\mathrm{a}}$, Tabinda Rasheed ${ }^{\mathrm{a}}$, Imtisal Ayman ${ }^{\mathrm{a}}$, Sara Ajmal ${ }^{\mathrm{a}}$, Abdul Rehman $^{\mathrm{b}}$, Imran Shakir ${ }^{c}$, Philips O Agboola ${ }^{\mathrm{d}}$, Muhammad Farooq Warsi ${ }^{\mathrm{a}, *}$

${ }^{a}$ Department of Chemistry, The Islamia University of Bahawalpur, Bahawalpur, 63100, Pakistan

${ }^{b}$ Department of Chemistry, Govt. College University Faisalabad, Faisalabad, 38000, Pakistan

c Sustainable Energy Technologies Center, College of Engineering, King Saud University, POBOX 800, Riyadh 11421, Saudi Arabia

${ }^{d}$ College of Engineering Al-Muzahmia Branch, King Saud University, P.O. Box: 800, Riyadh, 11421, Saudi Arabia 
*Author to whom the correspondence should be addressed: Email: farooq.warsi@iub.edu.pk

\section{Different approaches to synthesize MXene}

Method 1 (HF treatment):

- As described in the manuscript.

Method 2 (HCL/LiF treatment):

1. Etchant solution was prepared by mixing $1.6 \mathrm{~g} \mathrm{LiF}$ and $15 \mathrm{~mL} \mathrm{HCl}(37 \mathrm{wt}$. \%) into $5 \mathrm{~mL}$ of DI water in a Teflon vessel.

2. Teflon vessel was used to avoid the risks owing to the in-situ production of HF.

3. $1 \mathrm{~g}$ of MAX powder was added gradually into the etchant mixture, placed in a fume hood, while continuous stirring.

4. Stirring was continued for $40 \mathrm{hrs}$ at room temperature.

5. The resultant product was washed with DI water and centrifuged for $3 \mathrm{~min}$. at $3500 \mathrm{rpm}$.

6. The precipitates obtained was then washed repeatedly until the $\mathrm{pH}$ of the supernatant was becomes $>6$.

7. The suspension was again centrifuged for $30 \mathrm{~min}$. at $3500 \mathrm{rpm}$.

8. The as-synthesized product was then vacuum filtered and dried carefully in an oven.

\section{Method 3 (Hydrothermal method):}

With NaOH: To prepare the etchant solution, DI water was boiled and treated with argon gas for $30 \mathrm{~min}$.

1. Calculated amount of $\mathrm{NaOH}$ was added in this water to make $27.5 \mathrm{M} \mathrm{NaOH}$ solution.

2. In a $50 \mathrm{~mL}$ autoclave, $25 \mathrm{~mL}$ of etchant solution along with $100 \mathrm{mg}$ of MAX powder was taken.

3. The autoclave was sealed and subjected to hydrothermal reaction at $270{ }^{\circ} \mathrm{C}$ for $12 \mathrm{hrs}$.

4. After $12 \mathrm{hrs}$, the resultant blackish suspension was filtered with PVDF membrane along with washing with DI water for various times.

5. Obtained precipitates were dried at $70{ }^{\circ} \mathrm{C}$ for $12 \mathrm{hrs}$ in a vacuum oven.

\section{With KOH:}

1. MAX powder $(0.1 \mathrm{~g})$ together with $\mathrm{KOH}(0.35 \mathrm{~g})$ was ground to a fine powder using pestle mortar.

2. A few drops of solvent (water, $0.05 \mathrm{~mL}$ ) were used to get a paste-like consistency.

3. As-prepared paste was transferred into a polytetrafluoroethylene-lined stainless-steel autoclave where the hydrothermal reaction was started at $180^{\circ} \mathrm{C}$ for $24 \mathrm{hrs}$.

4. After the required time for reaction, a solid product was obtained which was collected and washed with DI water repeatedly to neutralize the resultant solution.

5. The as-synthesized product was dried at $70{ }^{\circ} \mathrm{C}$ for $12 \mathrm{hrs}$ in a vacuum oven. 


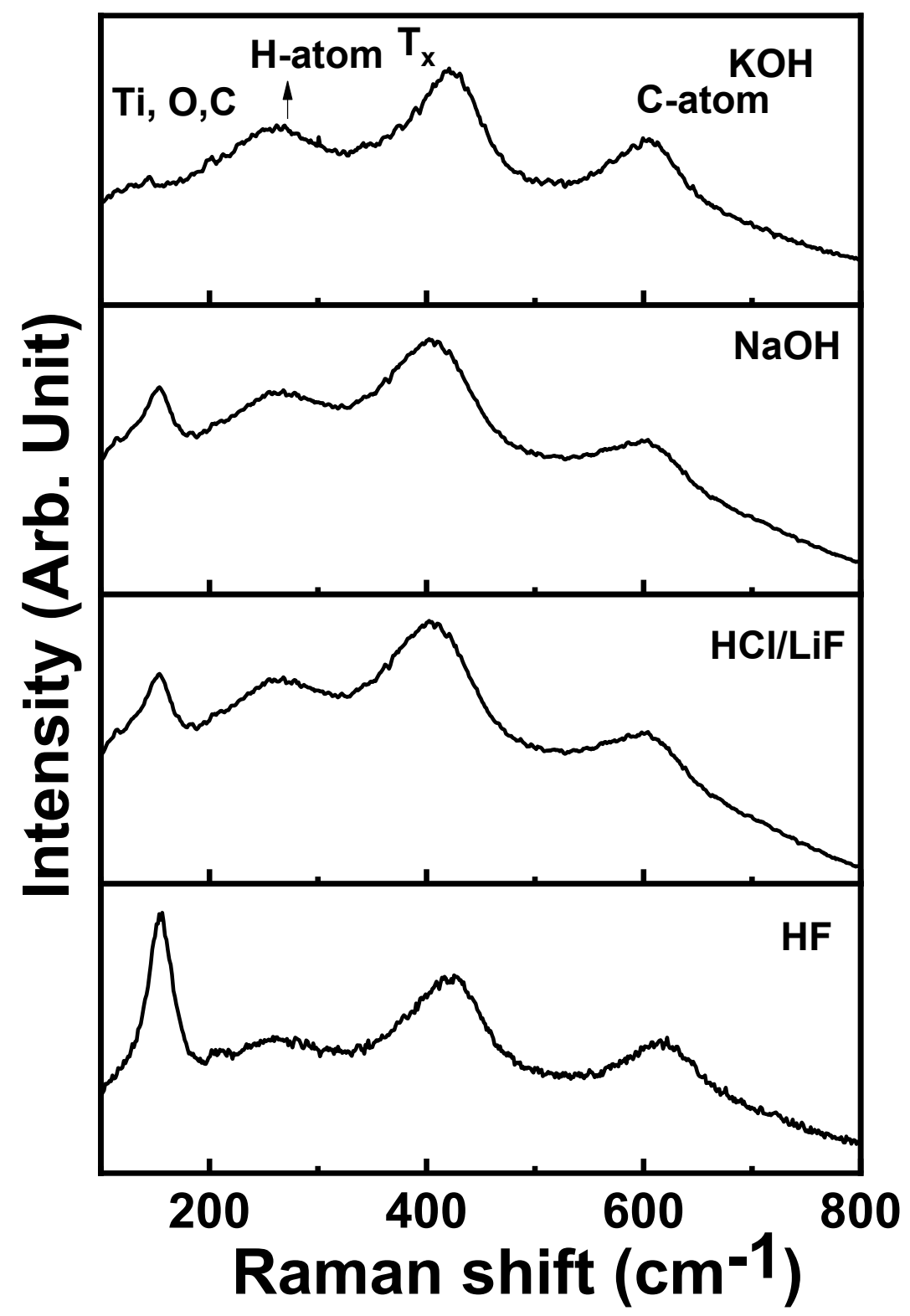

Figure S1: MXene synthesis using different synthesis approaches. 


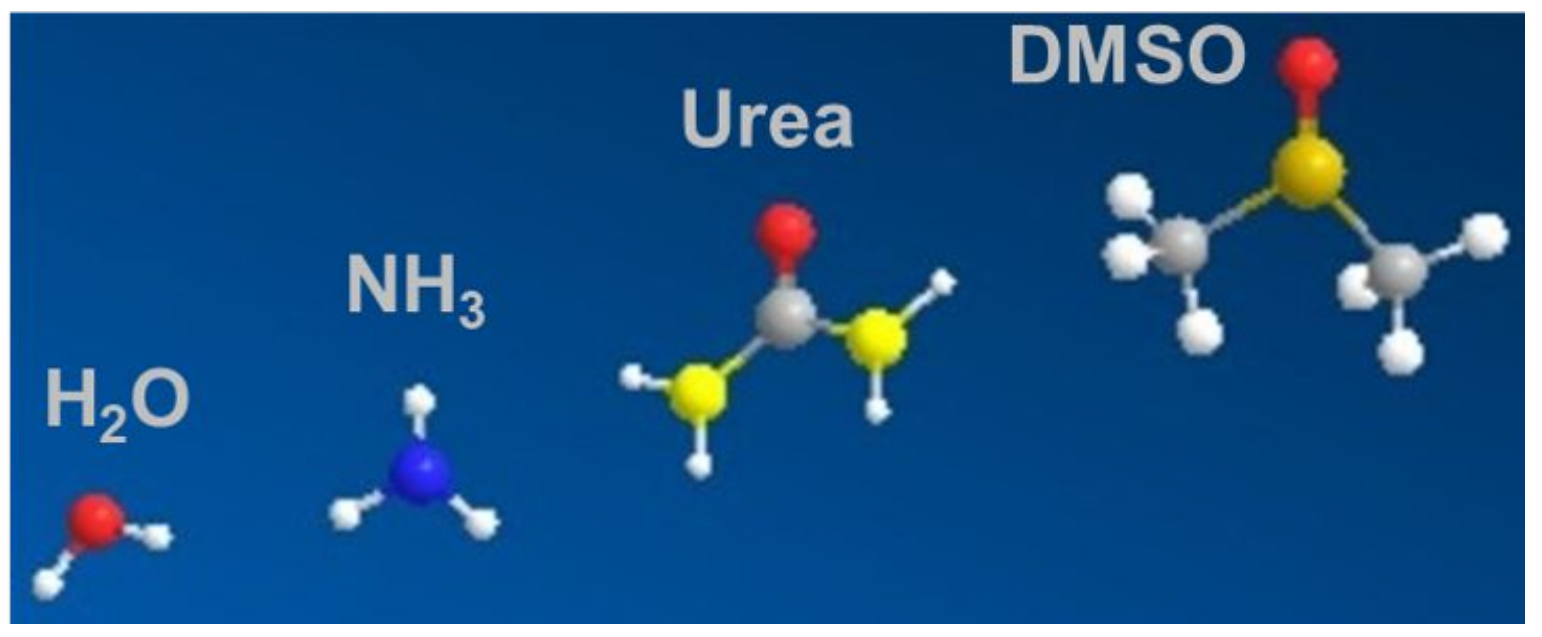

Figure S2: Molecular structure of used solvent molecules. 


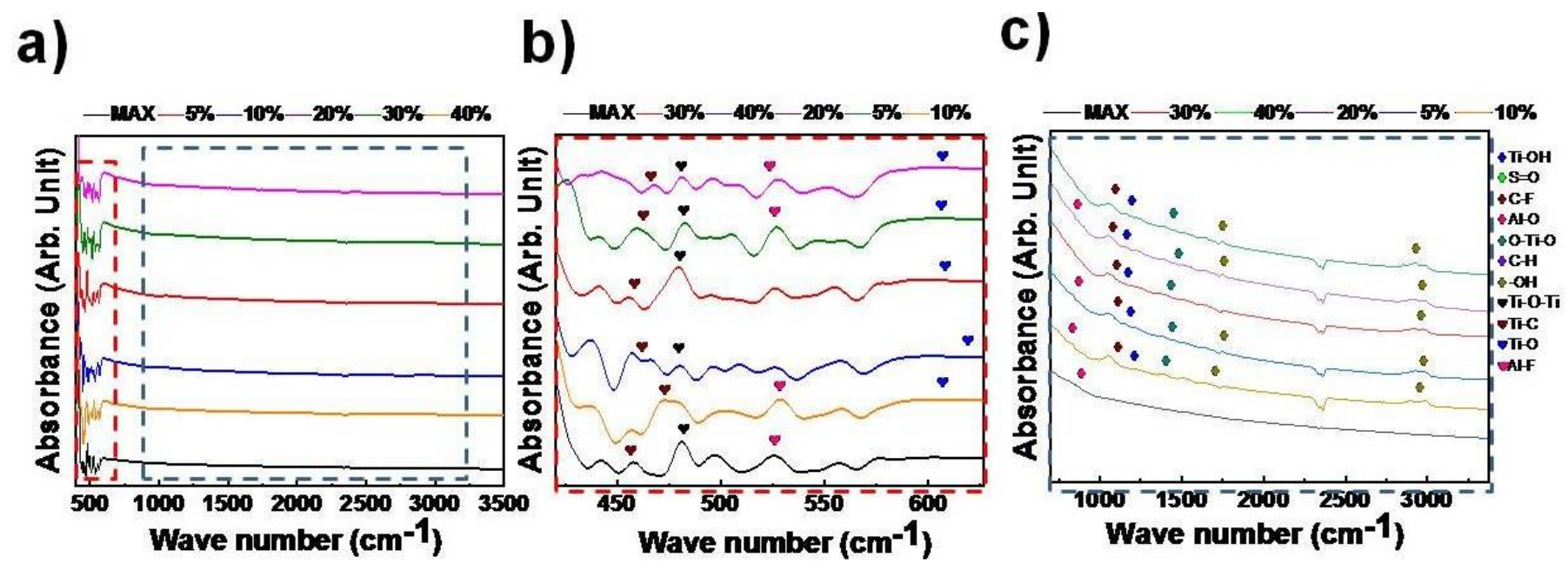

Figure S3: (a) FTIR spectrum of as-prepared MXene samples at different etchant concentrations (b) and (c) FTIR spectrum at specific scale to make all the absorption peaks prominent. 
a)

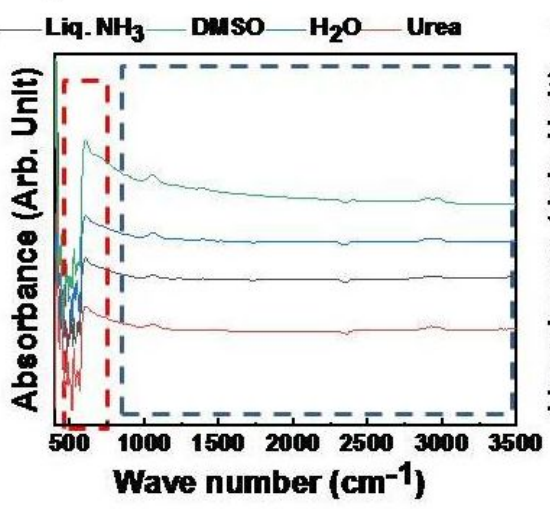

b)

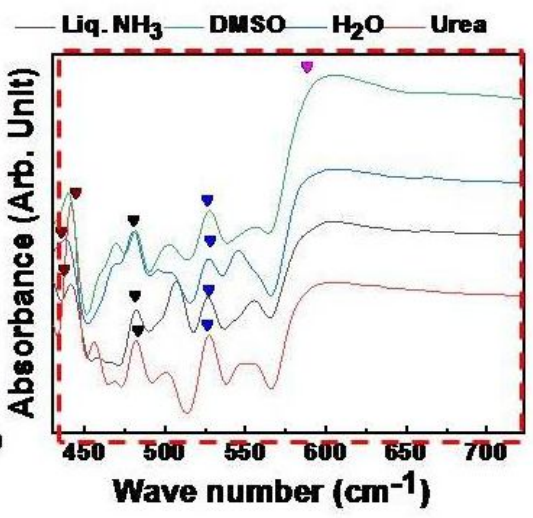

c)

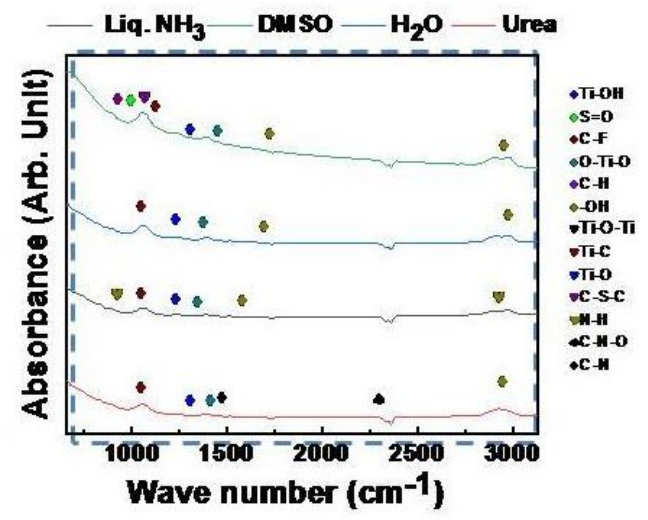

Figure S4 (a) FTIR spectrum of as-prepared MXene samples using different intercalants (b) and (c) FTIR spectrum at specific scale to make all the absorption peaks prominent. 

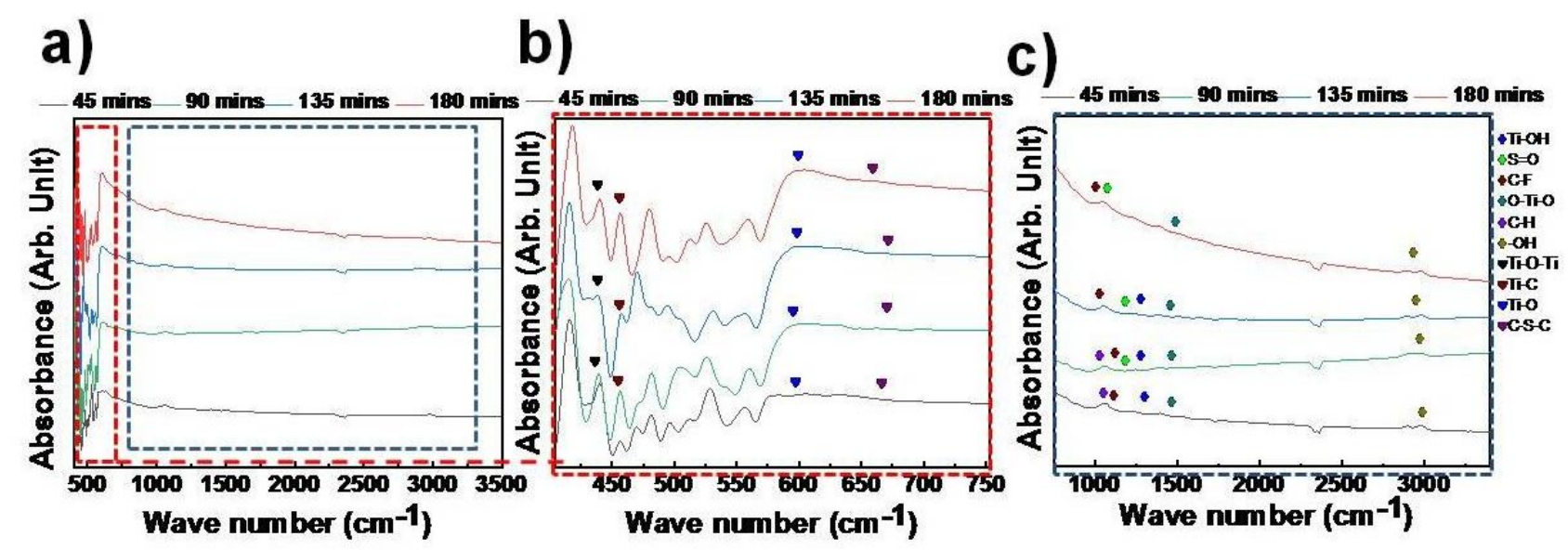

Figure S5: (a) FTIR spectrum of as-prepared MXene samples at different sonication times (b) and (c) FTIR spectrum at specific scale to make all the absorption peaks prominent. 
a)

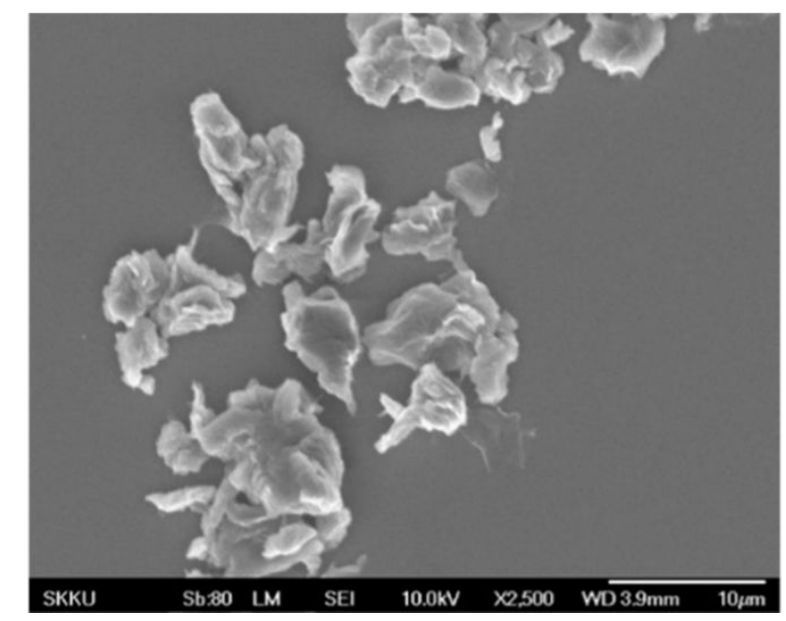

b)

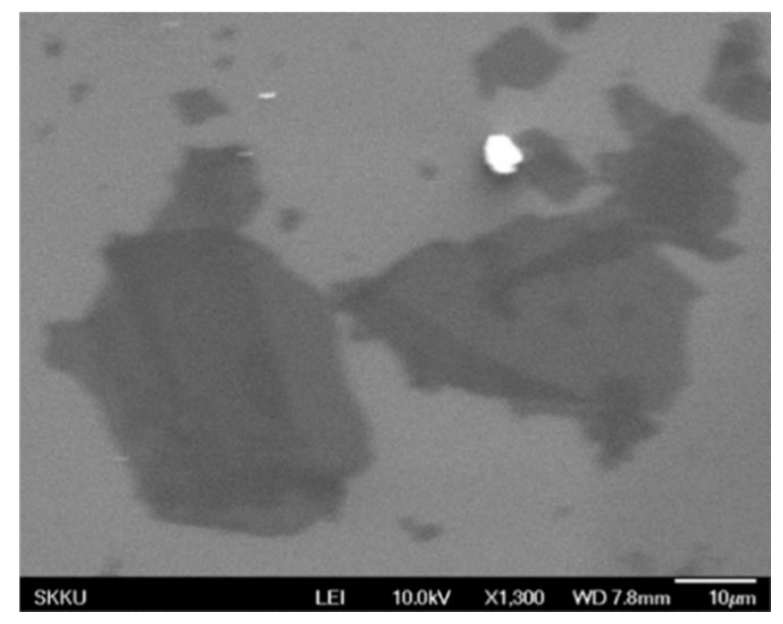

Figure S6: Delamination of Mxene (a) poor delamination (High Concentration of materials) (b) good delamination (Low Concentration of materials). 


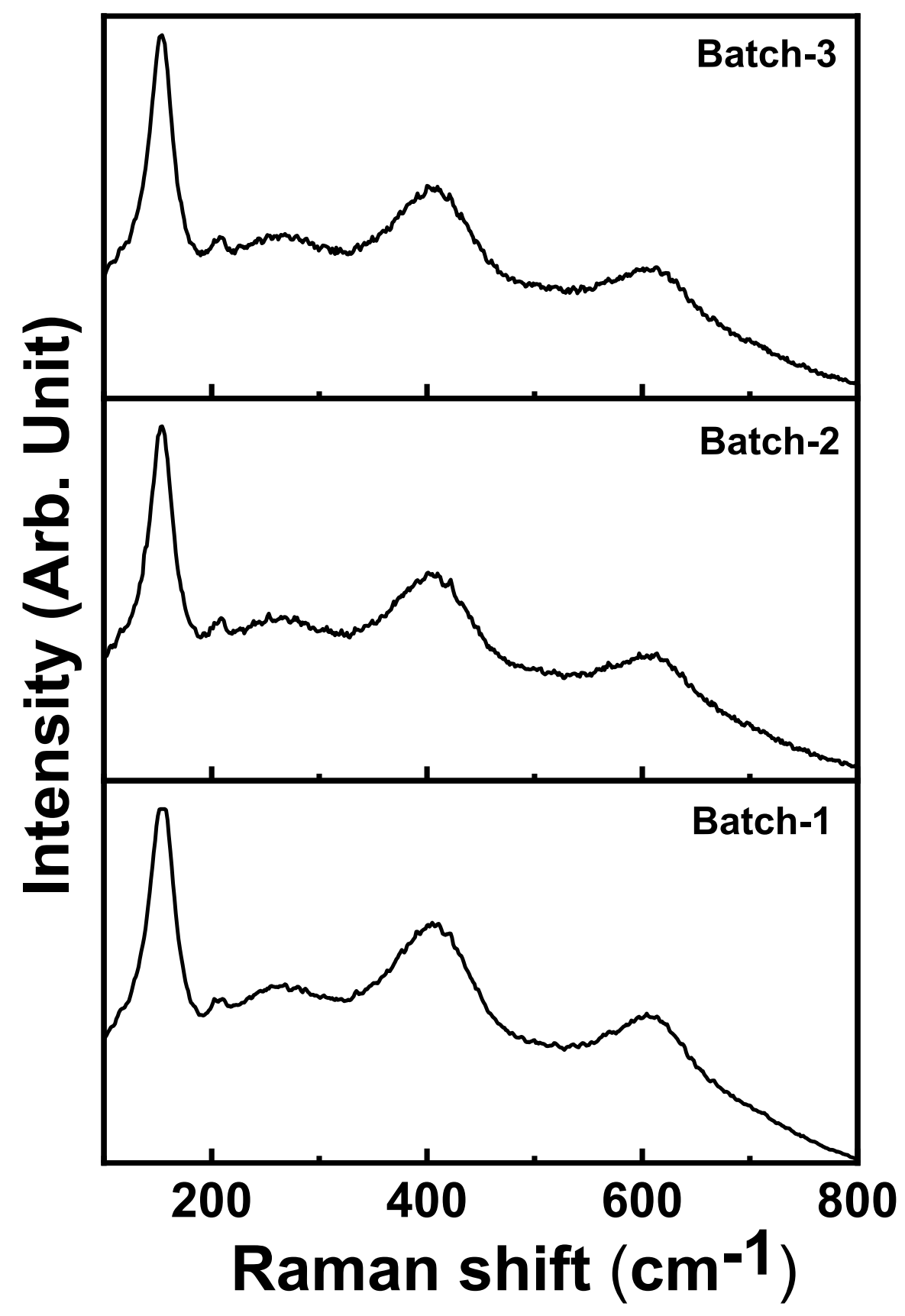

Figure S7: Reproducibility of the synthesized MXene. 
Table S1. Comparison of different intercalants with respect to their physical properties e.g. Boiling point, Molecular weight and dielectric constant b): 3D Molecular structure of intercalants.

\begin{tabular}{ccccc}
\hline Sr. No. & Intercalants & $\begin{array}{c}\text { Molecular } \\
\text { weight } \\
{[\mathbf{g} / \mathbf{m o l}]}\end{array}$ & $\begin{array}{c}\text { Boiling point } \\
{\left[{ }^{\circ} \mathbf{C}\right]}\end{array}$ & $\begin{array}{c}\text { Dielectric } \\
\text { constant } \\
{[\mathbf{F} / \mathbf{m}]}\end{array}$ \\
\hline $\mathbf{1}$ & DMSO & 78 & 189 & 46.7 \\
$\mathbf{2}$ & $\mathrm{Urea}$ & 60 & 132 & 2.9 \\
$\mathbf{3}$ & $\mathrm{H} 2 \mathrm{O}$ & 18 & 100 & 80 \\
$\mathbf{4}$ & Liq. $\mathrm{NH}_{3}$ & 17 & -33.34 & 31 \\
\hline
\end{tabular}


Table S2. Interlayer spacing of all the samples.

\begin{tabular}{lll}
\hline Sr. No. & Sample & d-spacing $(\boldsymbol{\mu m})$ \\
\hline $\mathbf{1}$ & MAX & -- \\
$\mathbf{2}$ & $5 \% \mathrm{HF}$ & 0.07 \\
$\mathbf{3}$ & $10 \% \mathrm{HF}$ & 0.18 \\
$\mathbf{4}$ & $20 \% \mathrm{HF}$ & 0.20 \\
$\mathbf{5}$ & $30 \% \mathrm{HF}$ & 0.26 \\
$\mathbf{6}$ & $40 \% \mathrm{HF}$ & 0.27 \\
$\mathbf{7}$ & Water & 0.21 \\
$\mathbf{8}$ & Aq.NH & 0.25 \\
$\mathbf{9}$ & Urea & 0.26 \\
$\mathbf{1 0}$ & DMSO & 0.29 \\
$\mathbf{1 1}$ & 45 mins & 0.33 \\
$\mathbf{1 2}$ & 90 mins & 0.34 \\
$\mathbf{1 3}$ & 135 mins & 0.49 \\
$\mathbf{1 4}$ & 180 mins & 0.50 \\
\hline
\end{tabular}


Table S3: Effect of different conditions on opening of layers of MXene.

\begin{tabular}{ccccccc}
\hline Sr. No. & $\begin{array}{c}\text { Conc. of } \\
\text { HF }[\mathbf{\%}]\end{array}$ & $\begin{array}{c}\text { Opening } \\
\text { of layers } \\
{[\boldsymbol{\mu m}]}\end{array}$ & $\begin{array}{c}\text { Intercalants } \\
{[\mathbf{1 5} \mathbf{~ m L}]}\end{array}$ & $\begin{array}{c}\text { Opening } \\
\text { of layers } \\
{[\boldsymbol{\mu m}]}\end{array}$ & $\begin{array}{c}\text { Sonication } \\
\text { time } \\
{[\mathbf{m i n s}]}\end{array}$ & $\begin{array}{c}\text { Opening } \\
\text { of layers } \\
{[\boldsymbol{\mu m}]}\end{array}$ \\
\hline $\mathbf{1}$ & 5 & 0.048 & $\mathrm{H}_{2} \mathrm{O}$ & 0.052 & 45 & 0.012 \\
$\mathbf{2}$ & 10 & 0.050 & $\mathrm{Aq} . \mathrm{NH}_{3}$ & 0.055 & 90 & 0.036 \\
$\mathbf{3}$ & 20 & 0.053 & Urea & 0.056 & 135 & 0.074 \\
$\mathbf{4}$ & 30 & 0.061 & DMSO & 0.063 & 180 & 0.064 \\
$\mathbf{5}$ & 40 & 0.060 & - & - & - & - \\
\hline
\end{tabular}


Table S4: Comparison with already reported literature.

\begin{tabular}{ccccc}
\hline $\begin{array}{c}\text { Etchants } \\
\text { (HF, } \\
\text { LiF+HCl, } \\
\text { KOH, }\end{array}$ & $\begin{array}{c}\text { Effect of } \\
\text { concentration } \\
\text { of etchant } \\
\text { (HF) }\end{array}$ & $\begin{array}{c}\text { Effect of } \\
\text { intercalants }\end{array}$ & $\begin{array}{c}\text { Effect of } \\
\text { sonication } \\
\text { time }\end{array}$ & Refrences \\
\hline$\checkmark$ & $\checkmark$ & $\checkmark$ & $X$ & 1 \\
$X$ & $X$ & $X$ & $X$ & 2 \\
$X$ & $X$ & $\checkmark$ & $X$ & 3 \\
$X$ & $X$ & $\checkmark$ & $X$ & 4 \\
$X$ & $X$ & $\checkmark$ & $X$ & 5 \\
$\checkmark$ & $\checkmark$ & $\checkmark$ & $\checkmark$ & This work \\
\hline
\end{tabular}




\section{Refrences}

(1) Alhabeb, M.; Maleski, K.; Anasori, B.; Lelyukh, P.; Clark, L.; Sin, S.; Gogotsi, Y. Guidelines

for synthesis and processing of two-dimensional titanium carbide (Ti3C2T x MXene). Chem. Mater 2017, 29, 7633-7644.

(2) Rajavel, K.; Ke, T.; Yang, K.; Lin, D. Condition optimization for exfoliation of two dimensional titanium carbide (Ti3C2T x). Nanotechnology 2018, 29, 095605.

(3) Mashtalir, O.; Naguib, M.; Mochalin, V. N.; Dall’Agnese, Y.; Heon, M.; Barsoum, M. W.; Gogotsi, Y. Intercalation and delamination of layered carbides and carbonitrides. Nat. commun 2013, 4, 1-7.

(4) Lukatskaya, M. R.; Mashtalir, O.; Ren, C. E.; Dall'Agnese, Y.; Rozier, P.; Taberna, P. L.; Naguib, M.; Simon, P.; Barsoum, M. W.; Gogotsi, Y. Cation intercalation and high volumetric capacitance of two-dimensional titanium carbide. Science 2013, 341, 1502-1505.

(5) Naguib, M.; Unocic, R. R.; Armstrong, B. L.; Nanda, J. Large-scale delamination of multilayers transition metal carbides and carbonitrides "MXenes". Dalton trans. 2015, 44, 9353-9358. 